\title{
TNF-alpha-induced microglia activation requires miR-342: impact on NF-kB signaling and neurotoxicity
}

\author{
João Paulo Brás (1,2,3, Joana Bravo ${ }^{1,3,4}$, Jaime Freitas ${ }^{1,2}$, Mário Adolfo Barbosa1,2,3, Susana Gomes Santos ${ }^{1,2,3}$,
} Teresa Summavielle (1) ${ }^{1,4,5}$ and Maria Inês Almeida (1) ${ }^{1,2,3}$

\begin{abstract}
Growing evidences suggest that sustained neuroinflammation, caused by microglia overactivation, is implicated in the development and aggravation of several neurological and psychiatric disorders. In some pathological conditions, microglia produce increased levels of cytotoxic and inflammatory mediators, such as tumor necrosis factor alpha (TNFa), which can reactivate microglia in a positive feedback mechanism. However, specific molecular mediators that can be effectively targeted to control TNF-a-mediated microglia overactivation, are yet to be uncovered. In this context, we aim to identify novel TNF-a-mediated micro(mi)RNAs and to dissect their roles in microglia activation, as well as to explore their impact on the cellular communication with neurons. A miRNA microarray, followed by RT-qPCR validation, was performed on TNF-a-stimulated primary rat microglia. Gain- and loss-of-function in vitro assays and proteomic analysis were used to dissect the role of miR-342 in microglia activation. Co-cultures of microglia with hippocampal neurons, using a microfluidic system, were performed to understand the impact on neurotoxicity. Stimulation of primary rat microglia with TNF-a led to an upregulation of Nos2, Tnf, and II16 mRNAs. In addition, ph-NFkB p65 levels were also increased. miRNA microarray analysis followed by RT-qPCR validation revealed that TNF-a stimulation induced the upregulation of miR-342. Interestingly, miR-342 overexpression in N9 microglia was sufficient to activate the NF-kB pathway by inhibiting BAG-1, leading to increased secretion of TNF-a and IL-1 $\beta$. Conversely, miR342 inhibition led to a strong decrease in the levels of these cytokines after TNF-a activation. In fact, both TNF-astimulated and miR-342-overexpressing microglia drastically affected neuron viability. Remarkably, increased levels of nitrites were detected in the supernatants of these co-cultures. Globally, our findings show that miR-342 is a crucial mediator of TNF-a-mediated microglia activation and a potential target to tackle microglia-driven neuroinflammation.
\end{abstract}

\section{Introduction}

In recent years, the immune system has been associated with pathological events occurring in several neurodegenerative and psychiatric disorders ${ }^{1}$. Accord-

Correspondence: Maria Inês. Almeida (ines.almeida@ineb.up.pt)

${ }^{1}$ i3S - Instituto de Investigação e Inovação em Saúde, University of Porto, Rua Alfredo Allen 208, 4200-135 Porto, Portugal

${ }^{2}$ INEB - Instituto de Engenharia Biomédica, University of Porto, Rua Alfredo Allen 208, 4200-135 Porto, Portugal

Full list of author information is available at the end of the article

Edited by B. Joseph ingly, growing evidence shows that some of the patients suffering from these disorders have chronic microglia activation and overproduction of pro-inflammatory cytokines, such as tumor necrosis factor alpha (TNF$\alpha$, which sustains several neuroinflammatory processes ${ }^{2}$.

Microglia are the largest population of immune cells in the central nervous system (CNS), corresponding to $5-15 \%$ of all adult brain ${ }^{3}$. Under physiological conditions, these cells are located within the brain parenchyma, where they are in direct contact with neural progenitors, neurons, and other glial cells (namely astrocytes and

\section{(c) The Author(s) 2020}

(c) (i) Open Access This article is licensed under a Creative Commons Attribution 4.0 International License, which permits use, sharing, adaptation, distribution and reproduction cc) in any medium or format, as long as you give appropriate credit to the original author(s) and the source, provide a link to the Creative Commons license, and indicate if changes were made. The images or other third party material in this article are included in the article's Creative Commons license, unless indicated otherwise in a credit line to the material. If material is not included in the article's Creative Commons license and your intended use is not permitted by statutory regulation or exceeds the permitted use, you will need to obtain permission directly from the copyright holder. To view a copy of this license, visit http://creativecommons.org/licenses/by/4.0/. 
oligodendrocytes $)^{4}$. When an injury or infection takes place, microglia are recruited to the site where they engulf invading pathogens and extracellular debris by phagocytosis, supporting the normal function and integrity of the brain $^{5}$. In response to an inflammatory stimulus, microglia secrete a number of molecules such as proteinases, nitric oxide, reactive oxygen intermediates and proinflammatory cytokines, including interleukin-1 beta (IL$1 \beta$ ), interleukin-6 (IL-6), and TNF- $\alpha$, .

TNF- $\alpha$ is a major Th1-class pro-inflammatory cytokine, produced by a variety of immune cells. In the CNS, activated microglia are one of the main sources of TNF- $\alpha^{8,9}$. TNF- $\alpha$ can bind to TNFR1(p55) and/or TNFR2(p75), activating downstream signaling pathways that mediate a wide variety of biological responses, including apoptosis, cell differentiation, proliferation, survival, and inflammation $^{10}$. At basal levels, TNF- $\alpha$ has an important role in brain development, particularly by influencing hippocampal development and function ${ }^{11}$. However, in certain pathological conditions, increased levels of this cytokine overactivate microglia, which then causes neuronal damage, such as demyelination and/or neuronal degeneration. Overactivated microglia release cytotoxic molecules, including TNF- $\alpha$, which is produced by a positive feedback mechanism of autocrine activation ${ }^{12,13}$. Although the basic mechanisms by which TNF- $\alpha$ activates microglia have been previously reported, the specific molecular mediators that can be effectively targeted to control TNF- $\alpha$-mediated microglia overactivation and neuroinflammation, are still to be uncovered.

In the last decade, microRNAs (miRNAs) have been extensively studied owing to their regulatory role in many pathological events ${ }^{14,15}$. miRNAs are short, single-stranded, non-protein coding RNAs, which regulate gene expression at the post-transcriptional level by inducing mRNA translation inhibition or degradation ${ }^{16}$. Importantly, miRNAs are highly conserved in mammalians ${ }^{17}$ and are crucial players in key cellular processes, such as inflammation, cell death, and differentiation. Remarkably, dysfunction in one single miRNA can concomitantly impair several biological functions by acting on distinct mRNA targets, as previously described by us ${ }^{16-20}$. In addition, miRNAs can be encapsulated into extracellular vesicles, and mediate cell-to-cell communication ${ }^{19,21}$. All these features make miRNAs attractive candidates for use as biomarkers and/or as therapeutic targets.

Herein, we investigated the role of miRNAs in TNF- $\alpha$ driven microglia activation, in order to identify potential therapeutic targets for microglia chronic activation and neuroinflammation. Specifically, we aimed to unravel how TNF- $\alpha$ impacted miRNAs expression, to identify which of these miRNAs are essential for microglia activation, ultimately determining their impact on the cross-talk between microglia and neurons.

\section{Materials and methods}

\section{Animal ethical disclosure}

All procedures to obtain primary cell cultures were conducted in accordance with European regulations (European Union Directive 2010/63/EU) and were approved by the i3S Animal Ethics Committee and the Portuguese regulatory entity-Direcção Geral de Alimentação e Veterinária (DGAV, ref 11769/2014-05-15 to TS). Animal facilities and the people directly involved in animal experiments (J.P.B. and J.B.) were also certified. All efforts were made to ensure minimal animal suffering, and to follow the principles of the 3Rs.

\section{Cell culture \\ Mixed glial cells}

In brief, newborn Wistar Han rats (P1-P2) were decapitated and brains separated from the skull. Cerebellum and meninges were carefully removed for efficient brain dissection. Dissected tissue was treated with DNase and trypsin (Sigma, USA) before being dissociated and plated. Rat brain-derived mixed glial cells were cultured in T75 poly-D-lysine (Sigma) coated flasks for 21 days. Culture was maintained in DMEM (Dulbecco's modified Eagle's medium; Gibco, USA) supplemented with $10 \%$ fetal bovine serum (FBS; Biowest, France).

\section{Primary microglia}

Primary microglia were isolated from rat mixed glial cultures using the shaking method, as previously described $^{22}$. Microglia were obtained after 14 and 21 days of culture by shaking the flasks at $37^{\circ} \mathrm{C}$ for $2 \mathrm{~h}$ at $150 \mathrm{rpm}$. Isolated microglia were re-seeded $\left(3 \times 10^{4} / \mathrm{cm}^{2}\right)$ in DMEM/F12 (Corning, USA) supplemented with $10 \%$ FBS, allowed to adhere for $48 \mathrm{~h}$, and then stimulated for $6 \mathrm{~h}$ with $100 \mathrm{ng} / \mathrm{mL}$ lipopolysaccharide (LPS, Sigma) or $20 \mathrm{ng} /$ mL TNF- $\alpha$ (Peprotech, UK).

\section{Primary hippocampal neurons}

E17 C57BL/6 mice hippocampal neurons were cultured as previously described ${ }^{23}$. In brief, after dissection, hippocampi were treated with trypsin $(1.5 \mathrm{mg} / \mathrm{mL}, 15 \mathrm{~min}$, $37^{\circ} \mathrm{C}$, Sigma) in Hank's balanced salt solution (HBSS; Gibco), washed with HBSS containing 10\% FBS, to stop trypsin activity, and washed in HBSS to remove serum and avoid glia growth. Finally, the tissue was transferred to serum-free Neurobasal medium (Gibco), supplemented with B27 (1:50, Gibco), glutamine (0.5 mM, Sigma), gentamycin $(0.12 \mathrm{mg} / \mathrm{mL}$ Gibco), and glutamate $(25 \mu \mathrm{M}$, Sigma), and dissociated mechanically. Neurons were then plated $\left(1 \times 10^{5}\right.$ cells/chamber $)$ in poly-D-lysine-coated coverslips $\left(20 \mu \mathrm{g} / \mathrm{cm}^{2}\right)$ previously attached to Axon Investigation Systems (AXIS, AX150, Millipore) and maintained in the supplemented neurobasal medium. Cells were kept at $37^{\circ} \mathrm{C}$ in a humidified incubator with $5 \%$ 
$\mathrm{CO}_{2} / 95 \%$ air, for 14 days, and half of the media was replaced at day 7 .

\section{N9 microglial cells}

Murine N9 microglia cells, kindly donated by professor João Relvas (i3S, Porto), were cultured in RPMI 1640 (Corning, USA) supplemented with 10\% FBS in T75 flasks. All cell lines were tested and negative for mycoplasma contamination. Prior to transfections, cells were trypsinized, re-seeded $\left(1.5 \times 10^{4} / \mathrm{cm}^{2}\right)$ and allowed to adhere for $24 \mathrm{~h}$. $70 \%$ confluent N9 microglia cells were transfected with mirVana miRNA mimic/mirVana miRNA inhibitor for mmu-miR-342-3p, or respective controls (mirVana miRNA mimic/inhibitor negative controls; Invitrogen, USA), using Lipofectamine 2000 (Invitrogen), as recommended by the manufacturer. After transfection, when applicable, N9 microglia cells were stimulated with $20 \mathrm{ng} / \mathrm{mL}$ of TNF- $\alpha$ for $6 \mathrm{~h}$. Conditioned media was collected for cytokine quantification and cells harvested for protein extraction or co-culture with neurons. Transfections with siRNA were also performed with Lipofectamine 2000. siRNA specific for murine BAG-1 (5'-CCGUUGUCAGCACUUGGAAUGCAAA-3') and SiRNA negative control $(12,935-300)$ were purchased from Invitrogen. Overexpression of BAG-1 in N9 microglia was achieved by transiently transfecting the cells with a BAG-1 mammalian expression vector (pCMV6-BAG-1, Origene). In brief, 24 hours before transfection, cells were seeded into six-well cell culture plates at a density of $1.2 \times 10^{5}$ cells per well in regular growth medium. Transfections were performed with Lipofectamine 2000 according to the manufacturer's instruction (Invitrogen). In all, 48 hours after transfection, cells were harvested for the detection of ph-NF-kB p65 expression levels by western blot. The control vector pCMV6 was kindly donated by Mariana Santos (UnIGENe, i3S, Portugal).

\section{Primary hippocampal neurons and N9 microglial cells co- culture}

Co-cultures were performed in Axon Investigation System (Millipore). At day 13 of neuronal culture, transfected, TNF- $\alpha$ or non-stimulated N9 microglia cells $(0.2 \times$ $10^{5}$ cells) were added to each Axon Investigation System, in direct contact with axons for $24 \mathrm{~h}$. Culture media was then collected for nitrites quantification and cells were fixed prior to immunostaining.

\section{Flow cytometry}

Primary microglia culture purity was measured by flow cytometry using the following antibodies: mouse anti-rat CD11b/c-PE/Cy7 (BD Biosciences, USA) and mouse anti-rat CD45-FITC (ImmunoTools, Germany). Unlabeled microglia, mouse isotypes IgG2a-PE/Cy7 (BD
Biosciences) and IgG2a-FITC (Immunoools) were used as negative controls. Fluorescence was measured in a FACS Canto II flow cytometer (BD Biosciences) with BD FACS Diva software. Results were analyzed using FlowJo Software.

\section{RNA extraction}

Total RNA was extracted using TRIzol reagent (Invitrogen) according to the manufacturer's instructions. RNA concentration and purity were evaluated in a NanoDrop 1000 (Thermo Scientific). Ratios of 260/280 and $260 / 230 \mathrm{~nm}$ ranged between 1.9 and 2.1. RNA integrity was evaluated by agarose gel electrophoresis or by Experion automated electrophoresis system (BioRad, USA).

\section{Reverse transcription and real-time quantitative polymerase chain reaction}

For gene expression analysis, RNA was treated with TURBO DNA-free Kit (Invitrogen) and cDNA was synthesized using Random Hexamers (Invitrogen), dNTPs (Bioline) and SuperScript III Reverse Transcriptase (Invitrogen). qPCR was carried out in CFX96 Touch RealTime PCR Detection System (Bio-Rad, USA) using cDNA, primers and iQ SYBR Green Supermix (Bio-Rad). Oligonucleotides used for qPCR experiments are shown in Supplementary Table 1.

miR-146b-5p, miR-342-3p, miR-124-3p, and let-7i-5p expression was evaluated using TaqMan miRNA assays (Applied Biosystems). In brief, cDNA was synthesized using 30 ng of RNA as a template, gene-specific stem-loop Reverse Transcription primer, and the TaqMan miRNA reverse transcription kit (Applied Biosystems). qPCR was carried out in CFX96 Touch Real-Time PCR Detection System (Bio-Rad) using cDNA, TaqMan probe and SsoAdvanced Universal Probes Supermix (Bio-Rad). Small nuclear RNA U6 was used as reference gene. All runs were performed in duplicate. Relative expression levels from eight independent experiments were calculated using the quantification cycle $\left(\mathrm{C}_{\mathrm{q}}\right)$ method, according to MIQE guidelines ${ }^{24}$.

\section{miRNA microarray assay}

miRNA expression profile of TNF- $\alpha$-activated primary rat microglia was performed through ArrayStar, using a $\mu$ Paraflo mouse miRNA microarray, Array 19.0 (LC Sciences, USA). By adding a poly (A) tail to the 3' end with poly (A) polymerase, total RNA (2 $\mu \mathrm{g} /$ sample $)$ was extended, and then an oligonucleotide tag was ligated to the poly (A) tail for fluorescent dye staining subsequently. Prepared RNA was hybridized to a $\mu$ Paraflo microfluidic chip. Detection probes were synthesized in situ, based on miRBase v22.0 database (www.mirbase.org) ${ }^{25}$. Following hybridization, the Cy3 dye was bound to the oligo tag for 
staining through the microfluidic chip. Fluorescence images were acquired with a laser scanner and digitized with Array-Pro image analysis software. Data relative to the fluorescent intensity of a given hybridization target (signal) is presented in arbitrary units. Only miRNAs with average signals $\geq 20$ and $-0.2 \leq \log _{2}$ fold change to CTR $\geq$ 0.2 were considered for further evaluation (Supplementary Table 3).

\section{Western blot}

N9 microglia cells were harvested and washed twice with cold PBS before lysis in the presence of protease and phosphatase inhibitors. Cell lysates were centrifuged $\left(14,000 \mathrm{rpm}, 10 \mathrm{~min}, 4{ }^{\circ} \mathrm{C}\right)$ and total protein quantified using DC protein assay kit (Bio-Rad, USA). Protein samples were resolved by SDS-PAGE in reducing conditions and transferred to nitrocellulose membranes, which were blocked in a solution of 5\% BSA in TBS-Tween $0.1 \%$. Membranes were then probed using the following primary antibodies: anti-ph-NF-kB p65, anti-BAG-1, anti- $\alpha-$ Tubulin, and anti-glyceraldehyde 3-phosphate dehydrogenase (GAPDH). Appropriate secondary antibodies conjugated to horseradish peroxidase were used for signal detection. Antibodies manufacturers and respective dilutions are indicated in Supplementary Table 2. Protein expression levels were quantified using ImageLab. $\alpha$-Tubulin and GAPDH were used as normalizers.

\section{Analysis of nuclear NF-kB translocation by imaging flow cytometry}

Murine N9 microglial cells were stimulated with $20 \mathrm{ng} /$ $\mathrm{mL}$ of TNF- $\alpha$ for 10, 20, or 30 minutes or transfected with the miRNA mimic mmu-miR-342-3p and the miRNA mimic negative control (SCR), as described. Before harvesting, cells were washed twice with ice-cold $\mathrm{PBS}$ and fixed in $4 \%$ PFA. NF-kB staining with rabbit anti-mouse Phospho-NF-kB p65 (Ser536) (93H1) antibody (Cell Signaling Technologies) was performed in PBS with $0.1 \%$ Triton X-100 and 2\% FBS for 20 min on ice, followed by a 20 min incubation with anti-rabbit Alexa Fluor 488labeled secondary antibody (Thermo Fisher Scientific) in PBS with 2\% FBS. Nuclei were stained with $20 \mathrm{~mm}$ DRAQ5 (Biostatus) for 10 min before acquisition. Cells were acquired using an ImageStreamX Imaging flow cytometer with the INSPIRE software and equipped with an Extended Depth of Field filter (Amnis, EMD Millipore), at the Bioimaging Center for Biomaterials and Regenerative Therapies (b.IMAGE - i3S, Porto). Data analysis was performed with IDEAS software (Amnis, EMD Millipore). Fluorescence compensation was performed with single-stained samples. For each sample 30,000 cells were acquired, and $>10,000$ single, in focus, double positive for NF-kB and DRAQ5 cells were analyzed for NF-kB translocalization. The IDEAS software nuclear translocation wizard was used to determine the similarity coefficient between the NF-kB and DRAQ5 (nuclei) staining's. As described by AM Silva et $\mathrm{al}^{26}$, nuclear translocation was considered for NF-kB/ DRAQ5 similarity coefficients above 1 .

\section{Protein identification by Nanoscale Liquid Chromatography coupled to tandem Mass Spectrometry (nano LC-MS/MS)}

Protein identification and quantitation was performed by nano LC-MS/MS. This equipment is composed by an Ultimate 3000 liquid chromatography system coupled to a Q-Exactive Hybrid Quadrupole-Orbitrap mass spectrometer (Thermo Scientific, Germany). Samples were loaded onto a trapping cartridge for $3 \mathrm{~min}$ and further separated in a nano-C18 column at $300 \mathrm{~nL} / \mathrm{min}$. Data acquisition was controlled by Xcalibur and Tune software (Thermo Scientific). The mass spectrometer was operated in data-dependent ( $\mathrm{dd}$ ) positive acquisition mode alternating between a full scan $(\mathrm{m} / \mathrm{z} 380-1580)$ and subsequent HCD MS/MS of the 10 most intense peaks from full scan. Raw data were processed using Proteome Discoverer 2.2.0.388 software (Thermo Scientific). Protein identification was performed with Sequest HT search engine against the Mus musculus entries from the UniProt database.

\section{Enzyme-linked immunosorbent assay (ELISA)}

Supernatants of N9 microglial cells were collected and processed ( $\left.1500 \mathrm{rpm}, 10 \mathrm{~min}, 4^{\circ} \mathrm{C}\right)$. TNF- $\alpha$, Il- $1 \beta$, IL-6, MIP-2, IL-12, IL-10, and IL-4 levels were evaluated by ELISA, according to the manufacturer's instructions (ABTS ELISA Development Kit, PeproTech). Cytokine levels were measured in a plate reader at $405 \mathrm{~nm}$, with wavelength correction at $650 \mathrm{~nm}$. Cytokine concentrations $(\mathrm{pg} / \mathrm{mL})$ were determined using a standard calibration curve.

\section{Immunofluorescence}

Primary neurons and N9 microglial cells were washed and fixed with $4 \%$ paraformaldehyde (PFA) in PBS. Cells were permeabilized with $0.25 \%$ Triton in PBS prior to blocking and overnight incubation at $4{ }^{\circ} \mathrm{C}$ with primary antibodies: mouse anti- $\beta 3$-Tubulin (Biolegend) and rabbit anti-Iba1 (Wako) for neurons and microglia, respectively. Secondary antibodies anti-mouse Alexa 488 (Cell Signaling Technologies) and anti-rabbit Alexa 594 (Invitrogen) were incubated for $1 \mathrm{~h}$ at RT. Nuclear staining was performed by incubating cells with Hoechst (Sigma) for $5 \mathrm{~min}$ at RT. Coverslips were mounted in microscope 
slides with Fluoroshield (Sigma) and images randomly acquired in a Zeiss Axio Imager Z1 Apotome. Neuronal apoptosis was addressed by evaluating nuclei shape of ten images per condition ${ }^{27}$.

\section{Nitrites quantification (Griess assay)}

Supernatants from neuron-N9 microglia co-cultures were mixed with an equal volume of Griess reagent in a 96-well plate. Sodium nitrite (1000 nM, Sigma) was serial diluted to generate the standard curve. Absorbance was read at $550 \mathrm{~nm}$ and nitrites concentration calculated using a standard curve.

\section{Statistical analysis}

Statistical analysis was performed using GraphPad Prism version 7 (GaphPad Software, Inc.). Gaussian distribution was tested by the Shapiro-Wilk normality test. For non-normal distribution data, tests were used to evaluate significant differences between samples, namely Wilcoxon matched-pairs signed rank test (between two groups) and Friedman test, followed by uncorrected Dunn's multiple comparison test (more than two groups). When the data passed normality tests, one-way analysis of variance (more than two groups), followed by Sidak's multiple comparison test was used. The statistical test used is identified in each figure legend. Experiments were performed at least three times independently. All samples were included in the analysis. Statistical significance was considered for $p<0.05\left(" p<0.05,{ }^{* *} p<0.01,{ }^{* * * *} p<0.001\right.$, n.s.: non-significant).

\section{Results}

TNF- $\alpha$ induces microglia activation through NF-kB

To better understand the mechanism by which TNF- $\alpha$ activates microglia, primary rat mixed glial cells (microglia, oligodendrocytes, and astrocytes) were isolated from P1-P2 rats. Microglia was obtained using the well-described shaking method, which allowed the setup of microglial in vitro experiments with a cell purity $>99 \%$ (Supplementary Figs. 1 and 2). To assess the impact of TNF- $\alpha$ on the expression of inflammation-associated genes, microglia was stimulated with $20 \mathrm{ng} / \mathrm{mL}$ of recombinant TNF- $\alpha$ for $6 \mathrm{~h}$, determined as the peak of NF-kB p65 phosphorylation (Supplementary Figs 3). LPS $(100 \mathrm{ng} / \mathrm{mL})$ was used as a positive control of microglia activation. We observed that TNF- $\alpha$ significantly induced the upregulation of Nos 2 (mean fold change to CTR $(\mathrm{FC})=2.22, p=0.027), \operatorname{Tnf}(\mathrm{FC}=2.92, p=0.014)$ and $I l 1 b \quad(\mathrm{FC}=2.54, \quad p=0.026)$ pro-inflammatory mRNAs compared with non-stimulated microglia (Fig. 1a). Interestingly, TNF- $\alpha$ had no impact in the expression of Il-6, Il10 and Msr1 mRNAs, whereas LPS induced the upregulation of $I l 6(\mathrm{FC}=4.69, p=0.0016)$ and $I l 10$ $(\mathrm{FC}=2.28, p=0.027)$ and downregulation of $\mathrm{Msr} 1(\mathrm{FC}=$ $0.19, p=0.027$; Fig. 1a). Importantly, we also observed that TNF- $\alpha$-induced microglia activation resulted in

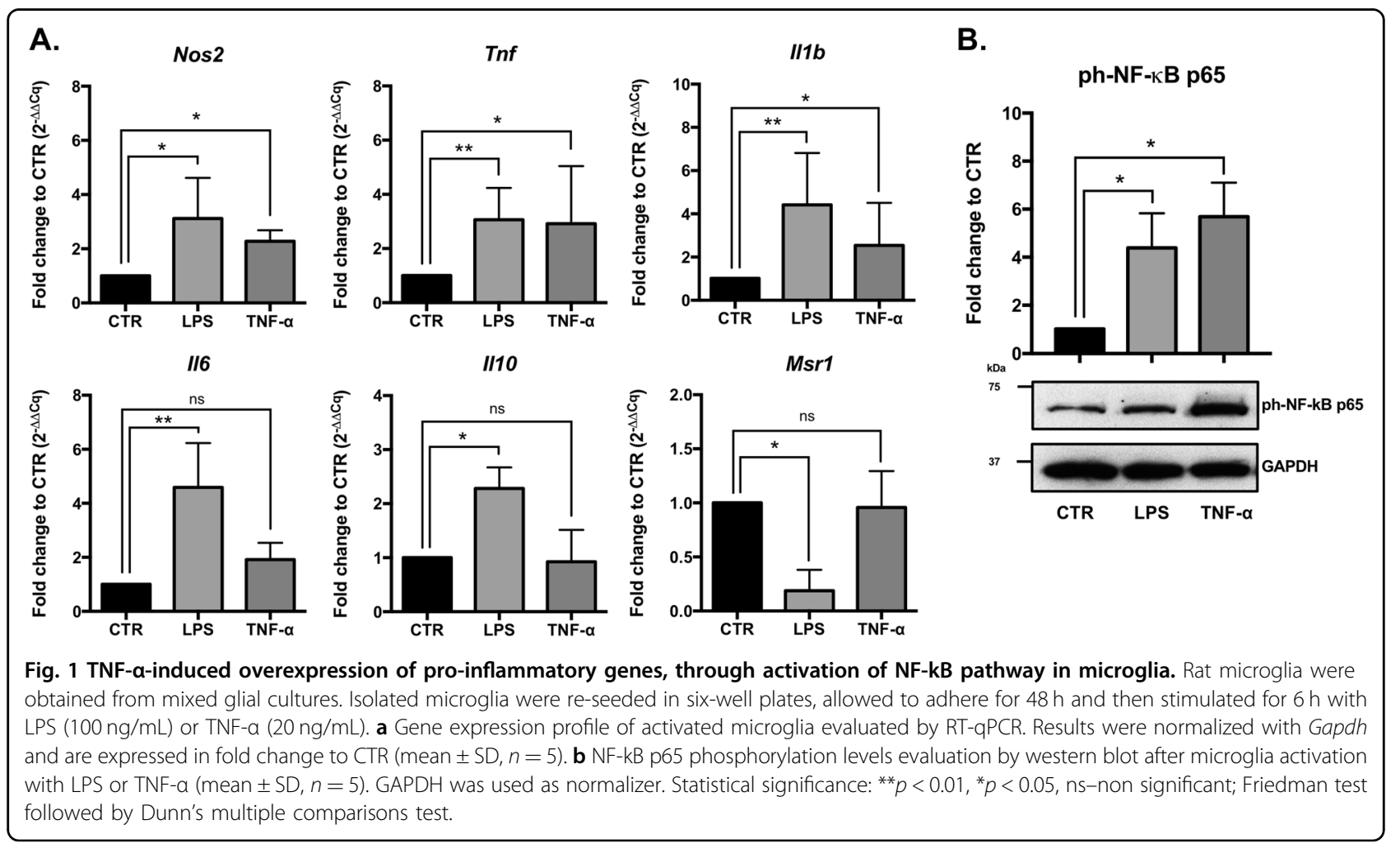


increased levels of ph-NF-kB p65 ( $p=0.011$; Fig. 1b), suggesting that the overexpression of the inflammatory mRNAs may be achieved through the activation of the NF-kB pathway.
miR-342 is overexpressed in TNF-a-activated microglia

In order to explore the role of miRNAs in TNF- $\alpha$-driven microglia activation, a miRNA microarray was performed in three independent experiments (Fig. 2a). Only miRNAs

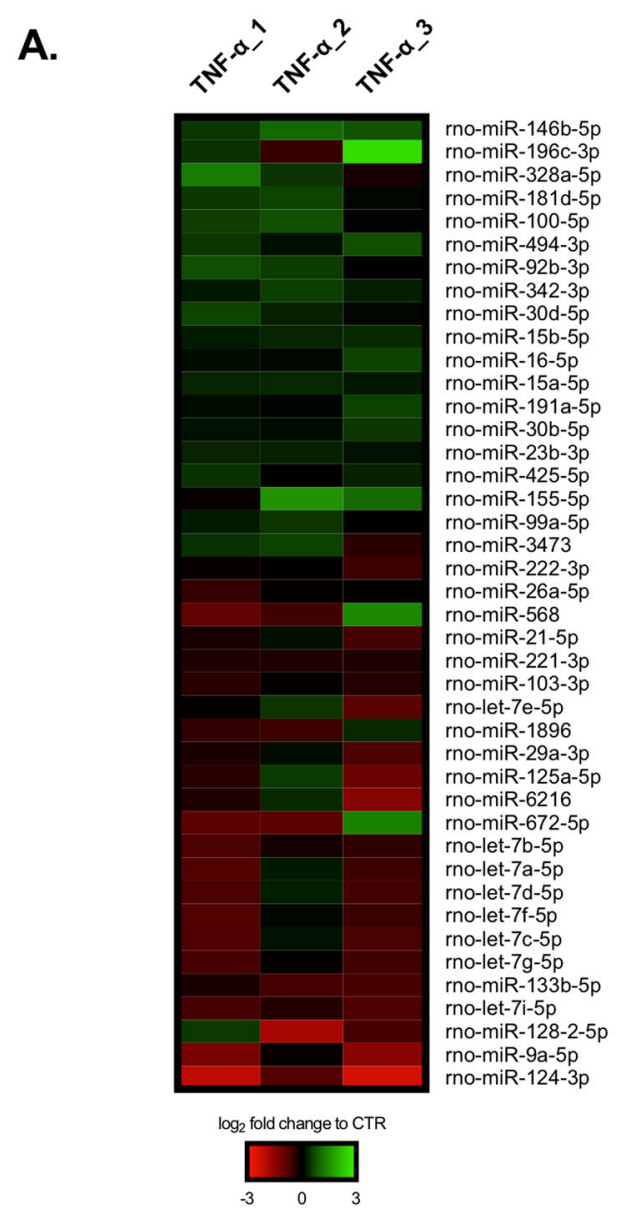

C. $\operatorname{miR-146b}$

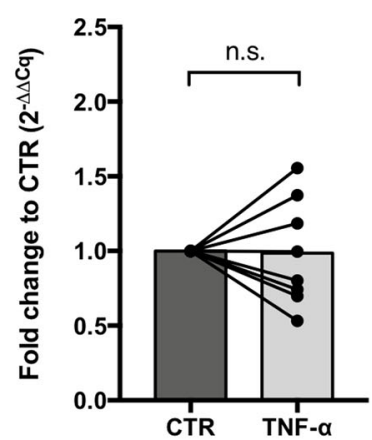

$\operatorname{miR}-342$

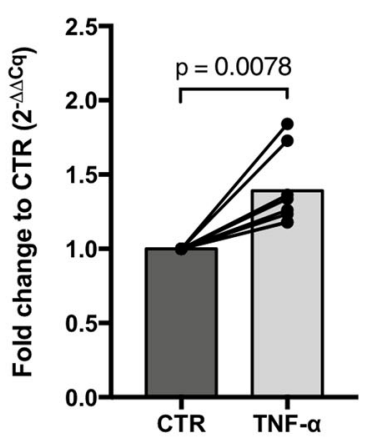

B.

\section{Top 10 upregulated miRNAs}

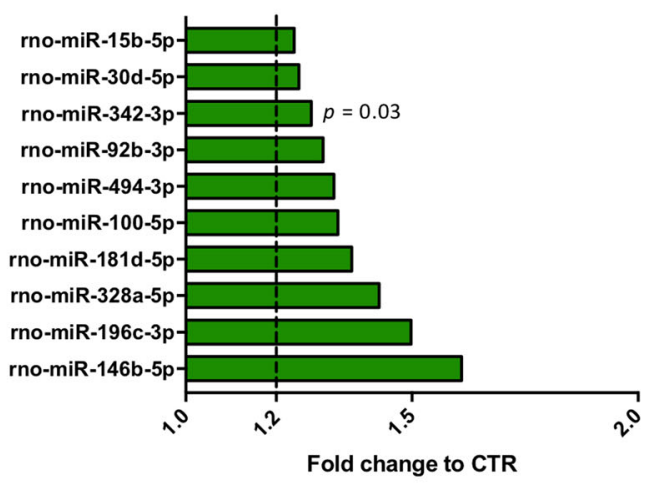

Top 10 downregulated miRNAs

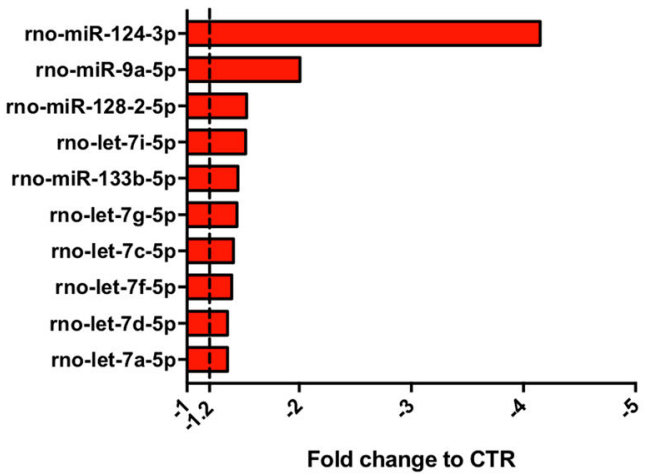

miR-124a

let-7i
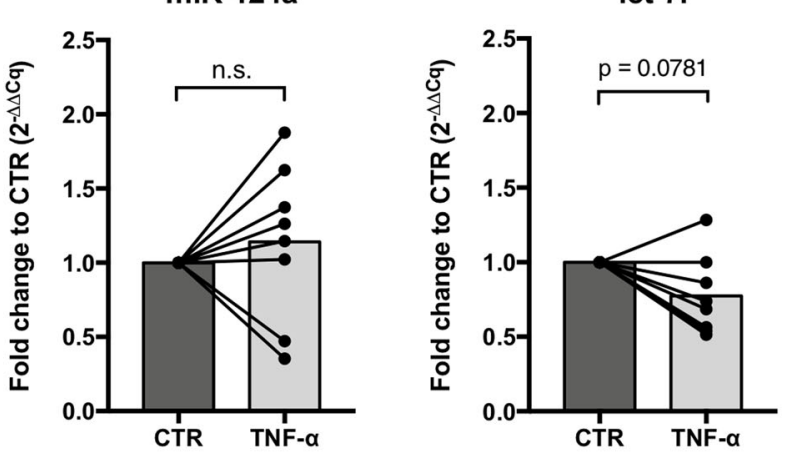

Fig. 2 miR-342 is overexpressed in TNF-a-stimulated microglia. a Heat map of miRNA microarray expression results in TNF-a activated microglia $(n=3)$. Only miRNAs with $-0.2 \leq \log _{2}$ FC to $C T R \geq 0.2$ and a detection signal $\geq 20$ are represented. $\mathbf{b}$ Ten most upregulated (green) or downregulated (red) miRNAs in TNF-a stimulated microglia (mean FC to CTR, $n=3$ ). c Fold change to control based on the relative expression of the selected miRNAs, evaluated by RT-qPCR (mean, $n=8$ ). U6 SnRNA was used as reference. Cq: quantification cycle. Statistical significance: $p<0.05$; Wilcoxon matched-pairs test. 
averaging a detection signal $>20$ were considered. Nineteen miRNAs were upregulated $\left(\log _{2} \mathrm{FC}\right.$ to $\left.\mathrm{CTR}>0.2\right)$, whereas 23 were downregulated $\left(\log _{2} \mathrm{FC}\right.$ to $\left.\mathrm{CTR}<-0.2\right)$ in microglia stimulated with TNF- $\alpha$ compared with control cells (Supplementary Table 3). miR-146b-5p was the most expressed miRNA (FC to CTR $=1.61$ ), however, only miR-342-3p was significantly upregulated (FC to $\mathrm{CTR}=1.28, p=0.03$; Fig. $2 \mathrm{~b}$ and Supplementary Table 3). miR-124-3p, the most downregulated miRNA (FC to $\mathrm{CTR}=-4.15$; Fig. $2 \mathrm{~b}$ and Supplementary Table 3), and several members of the let-7 family members were all downregulated on TNF- $\alpha$-activated microglia (Fig. $2 b$ ), although not statistically different to control. To validate these results, miRNA expression levels were evaluated by RT-qPCR. In fact, microarray results were confirmed, as only miR-342-3p was significantly upregulated after TNF- $\alpha$ stimulation (FC to CTR $=1.39 ; p=0.0078 ; n=8$ ). Neither miR-146b-5p, miR-124-3p, nor let-7i-5p (a member of let-7 family) were significantly altered (Fig. 2c). These results suggest that miR-342 may have a role in TNF- $\alpha$-mediated microglia activation.

miR-342 has a role in TNF-a-mediated microglia activation

To investigate the role of miR-342 in microglia activation following TNF- $\alpha$ stimulation, gain-of-function experiments were performed using the N9 microglia cell line (Supplementary Figure 4). Strikingly, we found that miR-342 overexpression per se, without TNF- $\alpha$ stimulation, was sufficient to increase ph-NF-kB p65 levels compared with non-stimulated $(p=0.004)$ and SCR-transfected microglia cells $(p=0.012$; Fig. $3 \mathrm{a})$. On the other hand, anti-miR-342 transfection resulted in a decrease in ph-NF-kB p65 levels compared with anti-SCR $(p=0.028)$, but not CTR (Fig. 3a). However, following TNF- $\alpha$ activation, miR-342 inhibition had a significant impact on the reduction of NF-kB p65 phosphorilation levels (vs TNF- $\alpha, p=0.044$; vs TNF- $\alpha+$ anti-SCR, $p=0.037$ ). The translocation of NF-kB to the nucleus under miR-342 overexpression was analyzed using ImageStreamX. Figure 3B shows a representative histogram of the similarity coefficient between NF-kB and nuclei of N9 microglia cells transfected with SCR (green) or miR-342 (yellow), and representative images of a cell with NF-kB in the cytoplasm (non-translocated) or colocalized with the nucleus (translocated). Transient transfection of N9 microglia cells with miR-342 resulted in a clear shift towards a higher similarity coefficient, which indicates that compared with SCR, miR-342 overexpression promotes NF-kB translocation to the nucleus. Quantification of the percentage of cells with nuclear localization of NF-kB shows a significant NF-kB nuclear translocation upon miR-342 overexpression (Fig. 3b). Although not significantly, the treatment with TNF- $\alpha$ also shows a tendency for inducing the translocation of NF-kB into the nucleus (Fig. 3b, $10 \mathrm{~min})$. The inflammatory role of miR-342 was further confirmed by the levels of pro-inflammatory cytokines produced by transfected microglia. Specifically, microglia overexpressing miR-342 show increased levels of TNF- $\alpha$ and IL-1 $\beta$, compared with non-stimulated (TNF- $\alpha, p=$ 0.021 ; IL-1 $\beta, p=0.008$ ) and SCR-transfected microglia cells (TNF- $\alpha, p=0.004$; IL-1 $\beta, p=0.022$; Fig. 4). Conversely, miR-342 overexpression had no effect on IL-6, MIP-2, IL12, IL-10, and IL-4. miR-342 inhibition, without TNF- $\alpha$ stimulation, had no impact on cytokine production compared with anti-SCR control (Fig. 4). In agreement with the RT-qPCR results shown in Fig. 1a, exposure to TNF- $\alpha$ induced the secretion of TNF- $\alpha(p=0.0006)$ and IL-1 $\beta(p<$ $0.0001)$, and also of MIP-2 $(p=0.019)$ compared with control microglia cells (Fig. 4), whereas no differences were detected for IL-6, IL-12, IL-10, and IL-4 (Fig. 4). Importantly, the increased secretion of TNF- $\alpha$, IL-1 $\beta$ and MIP-2 after TNF- $\alpha$ stimulation, was reduced by miR-342 inhibition (TNF- $\alpha, p=0.004$; IL-1 $\beta, p=0.004$; MIP- $2, p=0.027$, Fig. 4), which supports the hypothesis that miR-342 is needed for TNF- $\alpha$-driven microglia activation.

\section{miR-342 promotes NF-kB activation by inhibiting BAG-1}

Next, we searched for miR-342 target molecules and pathways/functions that could be involved in the TNF- $\alpha$ driven microglia activation. Thus, the protein expression profile of miR-342-transfected N9 microglia cells was analyzed (Fig. 5a, b). The nano LC-MS/MS identified 4399 proteins, of which 694 were downregulated (FC to $\mathrm{SCR}<$ -1.25 ) and 301 upregulated (FC>1.25; Fig. 5a, b and Supplementary Table 4). Interestingly, DAVID functional annotation $^{28}$, revealed that the group of upregulated proteins with the highest biological function enrichment score, are mainly involved in inflammatory responses (Fig. 5c). In the group of most downregulated proteins, we identified a potential candidate, BAG-1 ( $\mathrm{FC}=-1.42$; Fig. $5 \mathrm{~b})$. BAG-1 was previously described to degrade NF-kB p65, inhibiting inflammatory signaling in dendritic cells ${ }^{29}$. To validate these results, we carried out western blots against BAG-1 on proteins extracts from miR-342 and anti-miR342 transfected N9 microglial cells. Results show that miR-342 overexpression significantly inhibits BAG-1 expression (mean FC to $\mathrm{SCR}=0.546, p=0.031$; Fig. $6 \mathrm{a}$ ), whereas miR-342 inhibition induces an increase on BAG-1 expression (mean FC to anti-SCR $=1.911$, $p=0.078$; Fig. 6a). To confirm if NF-kB activation on miR-342-overexpressing N9 microglia cells occurs via BAG-1 inhibition, we used a siRNA targeting BAG-1 to inhibit its expression. Results clearly show that NF-kB p65 activation is dependent on BAG-1 expression, as its downregulation induced a significant overexpression of ph-NF-kB p65 (mean FC to siRNA NC $=4.18, p=0.012$; Fig. 6b). To further explore the role of BAG-1 on NF-kB p65 regulation, we transiently transfected N9 microglia cells with a BAG-1 mammalian expression vector. 

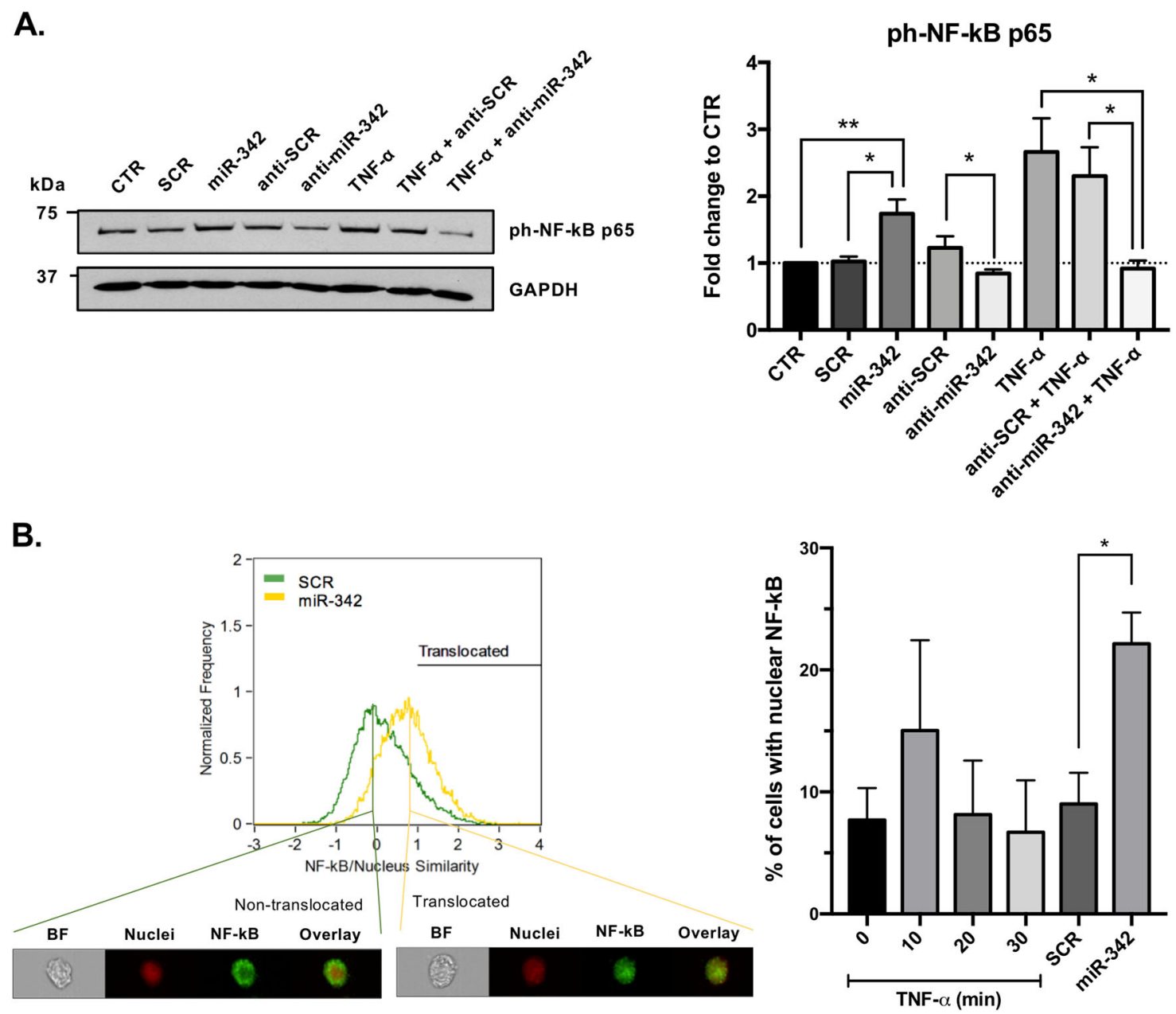

Fig. 3 miR-342 regulates TNF-a-mediated microglia activation through NF-kB. a ph-NF-kB p65 expression evaluation by western blot after TNFa stimulation and/or mirVana miRNA mimic/inhibitor mmu-miR-342-3p or mirVana miRNA mimic/inhibitor negative control (SCR) transfection. Results were normalized with GAPDH and compared with non-stimulated N9 microglia (mean \pm SD, $n=3-6$ ). $\mathbf{b}$ Representative plot of the similarity coefficient between NF-kB and nuclei staining's in cells transfected with mirVana miRNA mimic negative control (SCR, green) or miRNA mimic mmumiR-342-3p (miR-342, yellow). The black line (Translocated) corresponds to gated cells with nuclear translocated NF-kB (similarity coefficient $>1$ ). Representative images of cells with a similarity coefficient $<1$ (non-translocated) and with a similarity coefficient $>1$ (translocated) are shown below. $B F$ brightfield. On the right, graph shows the quantification of the percentage of cells with nuclear translocated NF-KB (translocated gate, similarity coefficient $>1$ ) after exposure to TNF-a for the indicated times or transfection with SCR or miR-342. Results are mean \pm SD of three independent experiments. ${ }^{*} p<0.05,{ }^{* *} p<0.01$; ANOVA followed by Sidak's multiple comparison test.

Although not significant, BAG-1 overexpression showed a trend towards a slight inhibition of NF-kB p65 phosphorylation levels compared with control (vs pCMV6, $p=0.08$ ). Nonetheless, it significantly inhibited the upregulation of ph-NF-kB induced by TNF- $\alpha$ (vs TNF- $\alpha$, $p=0.008$; vs TNF- $\alpha+$ pCMV6, $p=0.014$, Fig. $6 \mathrm{c}$ ). These results show that miR-342 promotes NF-kB activation by inhibiting BAG-1.

miR-342 overexpression in microglia induces neurotoxicity

After identifying the mechanism by which miR-342 participates on microglia activation, we addressed its functional consequences, specifically in the cross-talk with neurons.

Primary mouse hippocampal neurons were cultured on the right side of microfluidic chambers for 13 days, allowing axon projection through the device's microgrooves. Then, transfected/TNF- $\alpha$-stimulated N9 microglia cells were plated on the left side of the system (Fig. 7a and Supplementary Figure 5). After $24 \mathrm{~h}$ of co-culture, the supernatants were collected, and the cells were immunostained for the assessment of neuronal viability. Interestingly, TNF- $\alpha$-activated and miR-342-transfected microglia had a significant impact on neurons integrity 


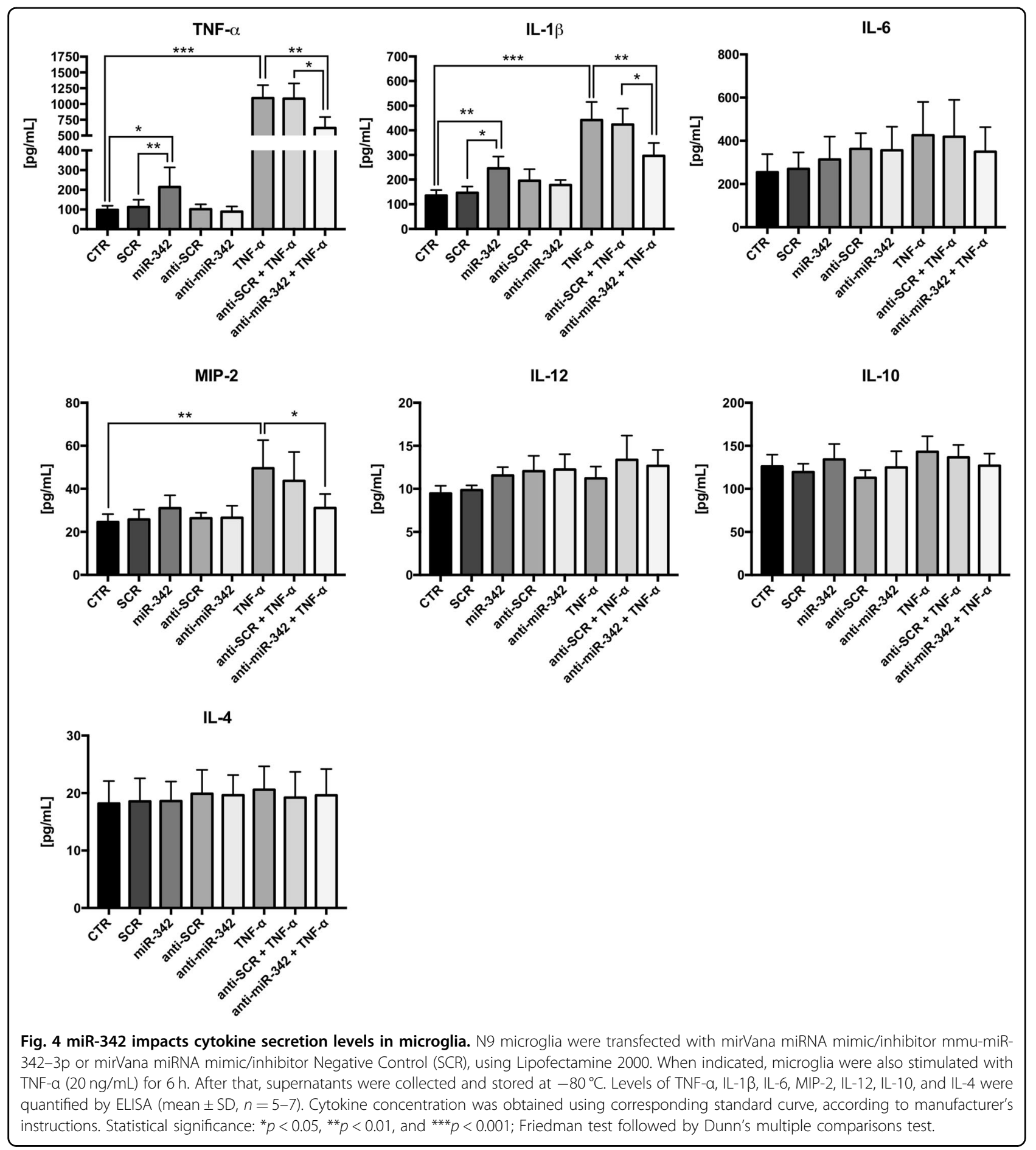

(Fig. 7b). TNF- $\alpha$ stimulated microglia significantly reduced neuron viability by $55 \%$, compared with nonstimulated microglia (FC to $\mathrm{CTR}=0.45, p=0.028$; Fig. 7c). miR-342-overexpressing N9 microglia cells also significantly induced neurotoxicity, reducing cell viability by $33 \%$ compared with CTR (FC to CTR $=0.67$, $p=0.041$, Fig. 7c) and in $38 \%$ compared with SCR- transfected microglia (FC to $\mathrm{CTR}=0.62, p=0.026$, Fig. 7c).

Considering that overactivated microglia increases the production of pro-inflammatory cytokines and nitric oxide species, which may have deleterious effect to the surrounding cells, we next determined nitrites concentration in the co-culture supernatants. In fact, the 
A.

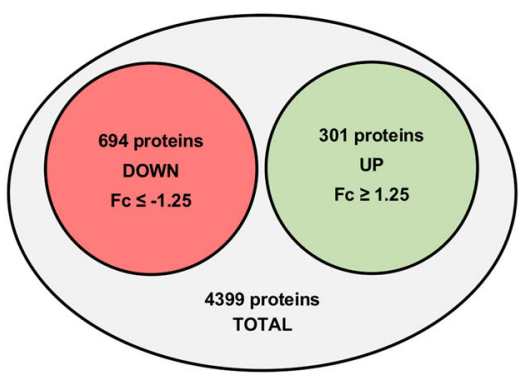

B.

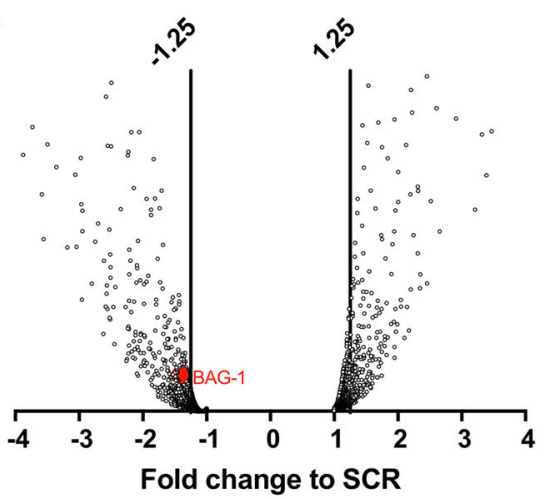

C.

\begin{tabular}{|c|c|c|c|}
\hline Enrichment Score: 1 & Proteins & Count & p-value \\
\hline Immune system process & $\begin{array}{l}\text { CD14, FADD, CR1L, HMGB2, LCN2, MIF, PTMS, } \\
\text { POLR3B, POLR3F, PSMB9, TLR13, TAP1 }\end{array}$ & 12 & $3.3 \times 10^{-2}$ \\
\hline Positive regulation of innate imune response & HMGB2, POLR3B, POLR3F & 3 & $5.3 \times 10^{-2}$ \\
\hline Innate imune response & $\begin{array}{l}\text { CD14, FADD, CR1L, HMGB2, LCN2, MIF, POLR3B, } \\
\text { POLR3F, TLR13, RELA }\end{array}$ & 10 & $7.9 \times 10^{-2}$ \\
\hline Defense response to virus & POLR3B, POLR3F, FADD & 3 & $6.9 \times 10^{-1}$ \\
\hline \multicolumn{4}{|c|}{ Most Downregulated Biological Functions ( $m i R-342$ vs $S C R, F C \leq-1.25$ ) } \\
\hline Enrichment Score: 0.46 & Proteins & Count & p-value \\
\hline Peptidyl-tyrosine phosphorilation & ABI3, JAK1, LYN, CSF1R, INSR & 5 & $2.3 \times 10^{-2}$ \\
\hline
\end{tabular}

Fig. 5 Protein expression profile of miR-342-transfected microglia. Protein identification and quantitation was performed by nano LC-MS/MS. Diagram $\mathbf{a}$ and volcano plot $\mathbf{b}$ represent the most differently expressed proteins within all identified proteins $(-1.25 \leq \mathrm{FC}$ to $S C R \geq 1.25, n=2)$. $\mathbf{c}$ Most up and downregulated biological functions based on protein expression fold change between miR-342 and SCR. Analysis was performed with DAVID using Functional Annotation Tool. The entire list proteins detected was used as background. "Enrichment score" indicates the biological relevance of the group of proteins involved in the respective set of biological functions, based on the $p$ values of all enriched annotation terms. "Count" indicates the number of dysregulated proteins involved in that specific biological function. Full protein names can be found in Supplementary Table 4.

quantification shows a significant increase in the levels of nitrites in the supernatants of both TNF- $\alpha$-stimulated $(p=0.0437)$ and miR-342-overexpressing $(p=0.0437)$ microglia co-cultures (Fig. $7 \mathrm{~d}$ ). These results support our hypothesis that miR-342-mediated microglia activation impacts the cross-talk with neurons(Fig. 8).

\section{Discussion}

Microglia are the primary immune effector cells in the CNS. Impairment of their normal structure or function, caused by either inflammatory activation (e.g., following infection, trauma, autoimmune, or neurodegenerative diseases) or by their decline and senescence (e.g., during aging or Alzheimer's disease), may cause damage in neuronal function and neurogenesis ${ }^{30-33}$. In addition, systemic alterations in the cytokine profile have been associated with distinct neurological and psychiatric diseases (e.g., depression, bipolar disorder, or schizophrenia) $)^{30,34,35}$. Pro- inflammatory cytokines, such as TNF- $\alpha$, a central mediator of neuroinflammation ${ }^{36}$, have access to the brain through humoral and neural routes, leading to microglia activation $^{37}$. In this work, we explored the impact of TNF- $\alpha$ on miRNAs expression in microglia and the mechanisms by which these miRNAs regulate cell activation. We show, for the first time, that miR-342 is a crucial mediator of TNF$\alpha$-driven microglia activation.

miR-342 was found upregulated in primary rat microglia activated with TNF- $\alpha$. To explore the role of miR-342 on TNF- $\alpha$-driven microglia activation we used a mouse cell line (N9), owing to the low (non-viral) transfection efficiency when working with primary microglia cells. Moreover, of the few microglia cell lines available, N9 is the one of the most commonly used and widely accepted. Importantly, the miR-342-3p sequence shows $100 \%$ homology between rat and mouse (Supplementary Figure 6). 

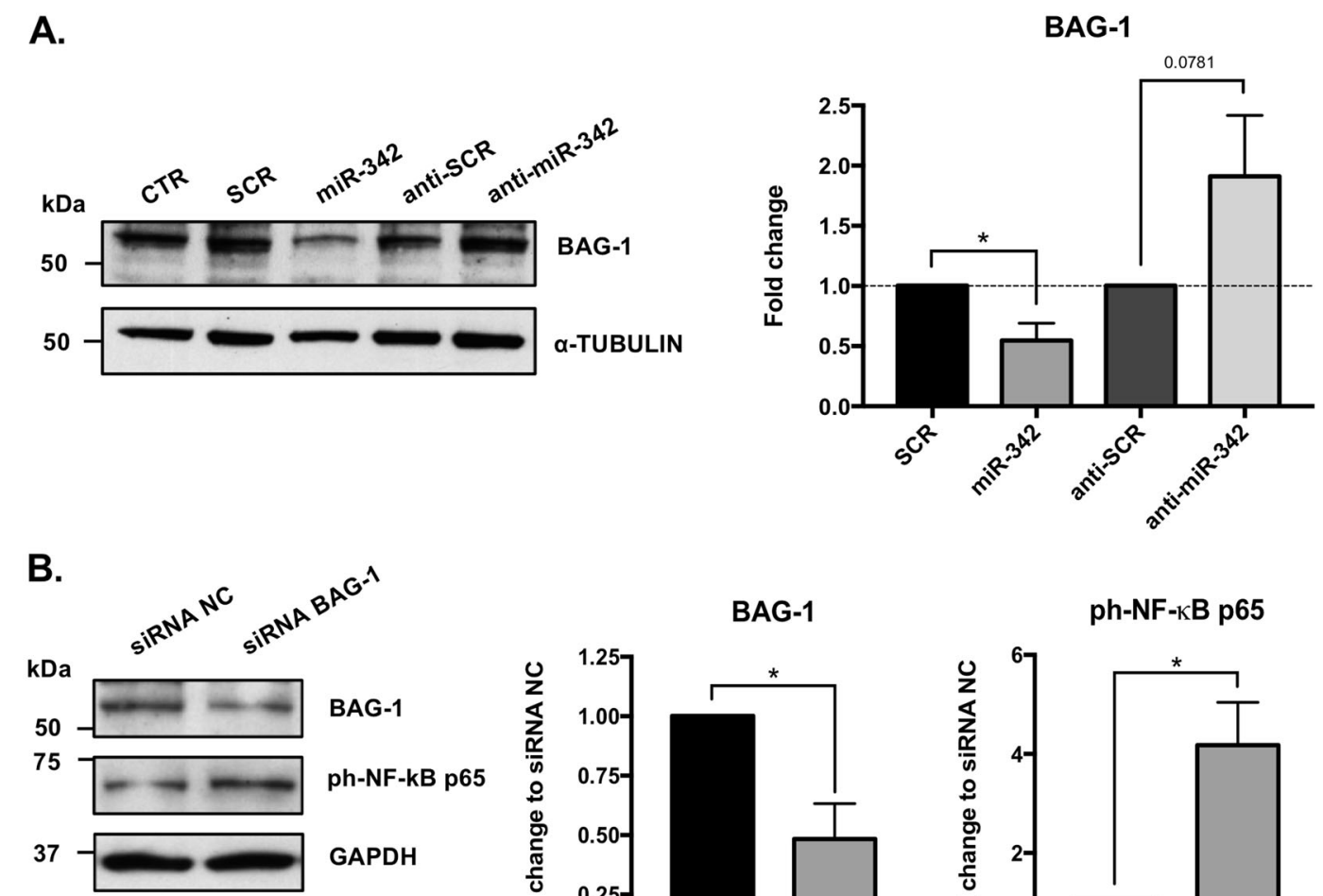

C.

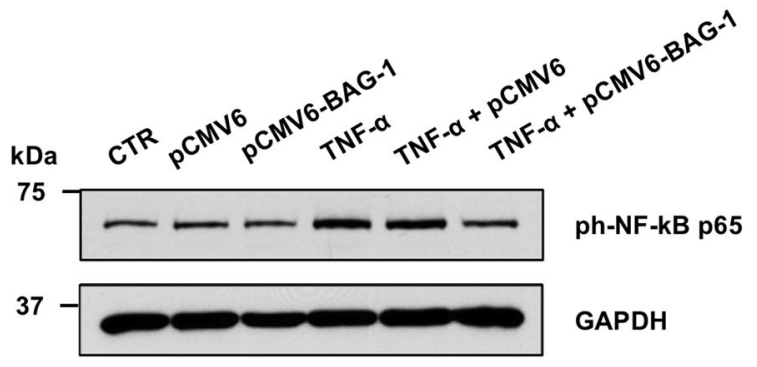

BAG-1

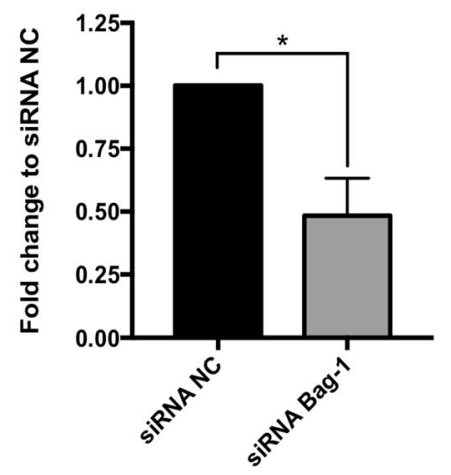

ph-NF-kB p65
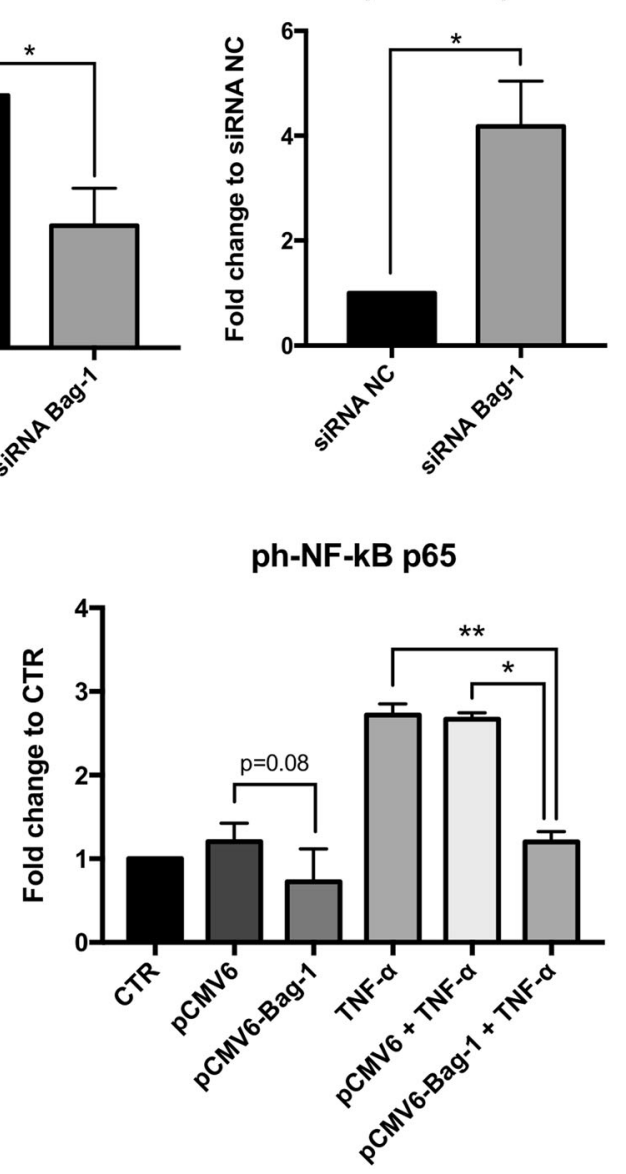

Fig. 6 miR-342 induces NF-kB activation by inhibiting BAG-1. a BAG-1 expression after miR-342 overexpression/inhibition was addressed by western blot. Results were normalized with a-tubulin and compared with the respective controls (mean \pm SD, $n=6$ ). To evaluate the involvement of BAG-1 on NF-kB activation, N9 microglia were transfected with a siRNA to silence $\mathbf{b}$ or with a plasmid (1ug/mL) to overexpress BAG-1 c. BAG-1 and ph-NF-kB p65 expression levels were evaluated by western blot. Results were normalized with GAPDH and compared with the respective controls (mean $\pm \mathrm{SD}, n=2-4$ ). Statistical significance: ${ }^{*} p<0.05$, Wilcoxon matched-pairs test.

Following miR-342 overexpression, we found that miR-342 per se, was sufficient to activate the NF-kB pathway, as shown by the increased ph-NF-kB p65 levels and NF-kB p65 nuclei translocation. Notably, miR-342driven NF-kB p65 activation led to increased secretion of TNF- $\alpha$ and IL-1 $\beta$. Conversely, miR-342 inhibition 
A.

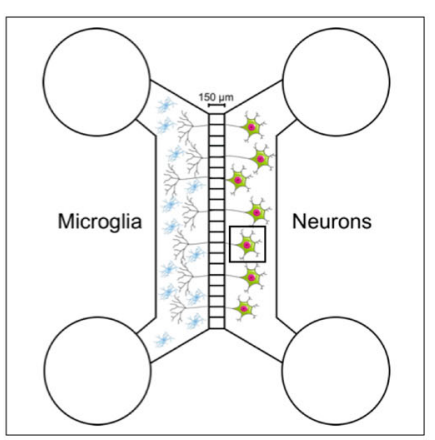

C.

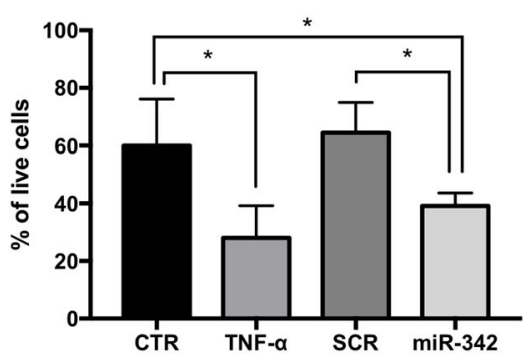

D.

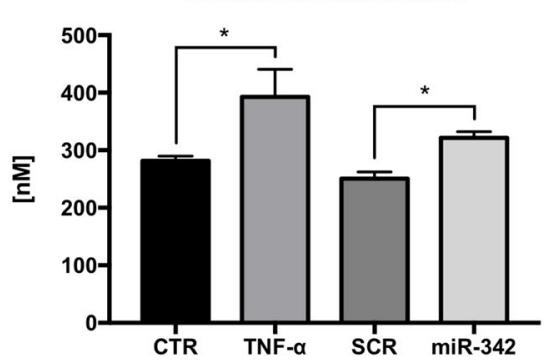

B.

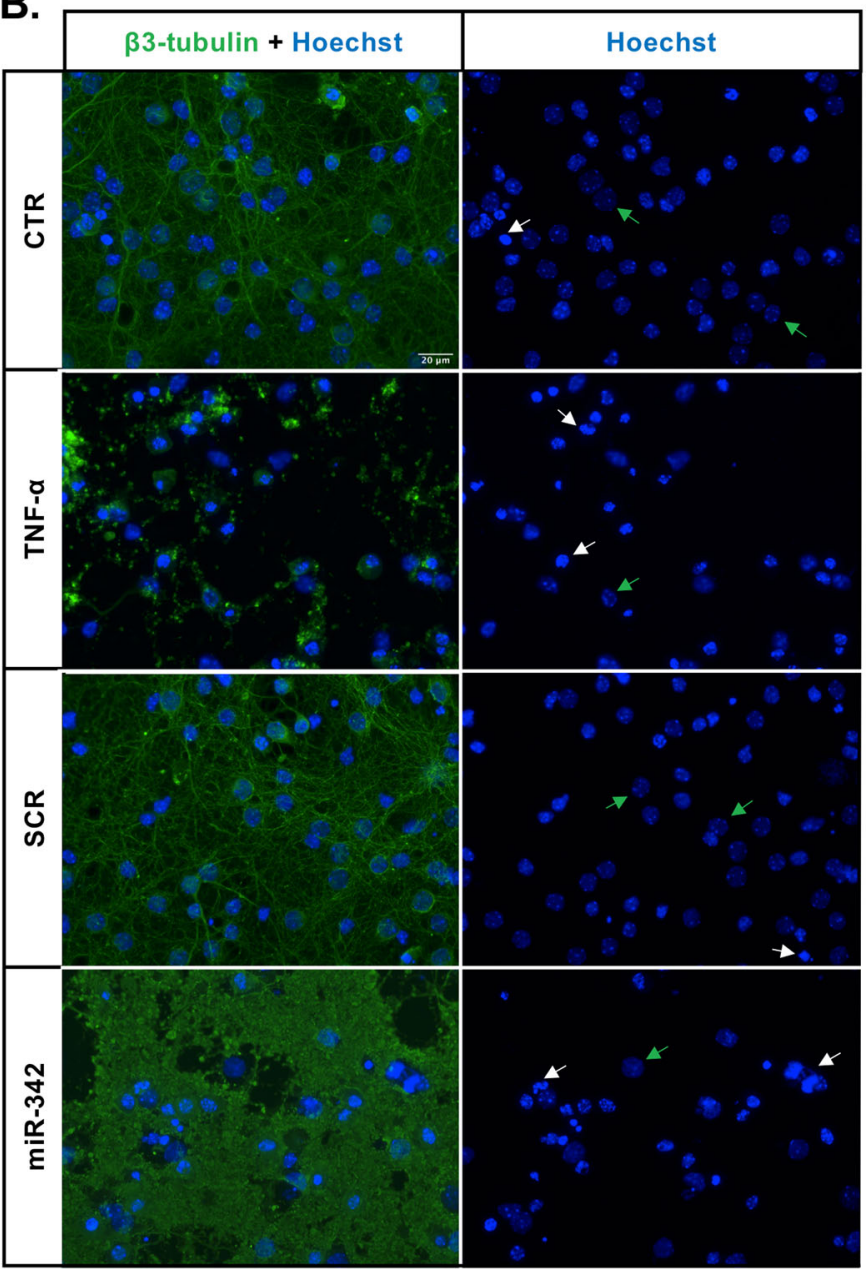

Fig. 7 miR-342 overexpression in microglia induces neurotoxicity. a Neurons were cultured in PDL-coated coverslips previously attached to the Axon Investigation System. At day 13 of neuronal culture, transfected, TNF-a, or non-stimulated N9 microglia were added to the respective system, in direct contact with axons for $24 \mathrm{~h}$. $\mathbf{b}$ Immufluorescence images of neurons after co-culture with N9 microglia. Left panel shows neurons stained with anti33 -tubulin (anti-Alexa 488, green) and Hoechst (blue). Right panel shows only Hoechst nuclear staining used for neuron viability evaluation. White arrows highlight the nucleus of dead neurons, whereas green arrows highlight the nucleus of healthy neurons. Scale bar: 20 um. c Neuron viability was addressed after counting the number of living and dead cells of 10 images per condition (mean $\pm \mathrm{SD}, n=4$ ). $\mathbf{d}$ Co-cultures' supernatants were collected for nitrite levels quantification using Griess reagent (mean \pm SD, $n=4$ ). Statistical significance: ${ }^{*} p<0.05$ and ${ }^{* *} p<0.01$, Friedman test followed by Dunn's multiple comparisons test.

strongly decreased the levels of these cytokines after TNF- $\alpha$ stimulation, suggesting that this miRNA is needed for microglia activation. Moreover, we observed that TNF- $\alpha$ mRNA and secreted levels were upregulated after TNF- $\alpha$ stimulation, in a positive feedback loop, possibly perpetuating neuroinflammation and ultimately promoting brain pathological conditions ${ }^{13}$. In line with our results, Kuno et al. ${ }^{13}$ proposed the microglia-derived TNF- $\alpha$ autocrine activation occurs via TNFR1 signaling pathway.

miRNAs act by degradation or translation inhibition of their target mRNAs ${ }^{16}$. Therefore, after observing that miR-342 overexpression increases the levels of ph-NF-kB p65, we hypothesized that this miRNA could be repressing an inhibitor of NF-kB. Consequently, we screened the LC-MS/MS data for proteins that were significantly downregulated and previously described as repressors of NF-kB. BAG-1 was the only candidate found to meet both criteria $^{29}$ (Supplementary Table 4). BAG-1 is known to interact with heat shock protein 70 (Hsp70) family of molecular chaperones, displaying a variety of cytoprotective activities and effects on signal transduction and transcription, suggesting a function in overcoming cellular stress and inflammation ${ }^{38,39}$. Tanaka et al. ${ }^{29}$ described that BAG-1, when associated with HSP70, can promote NF-kB p65 degradation in LPS-treated dendritic 


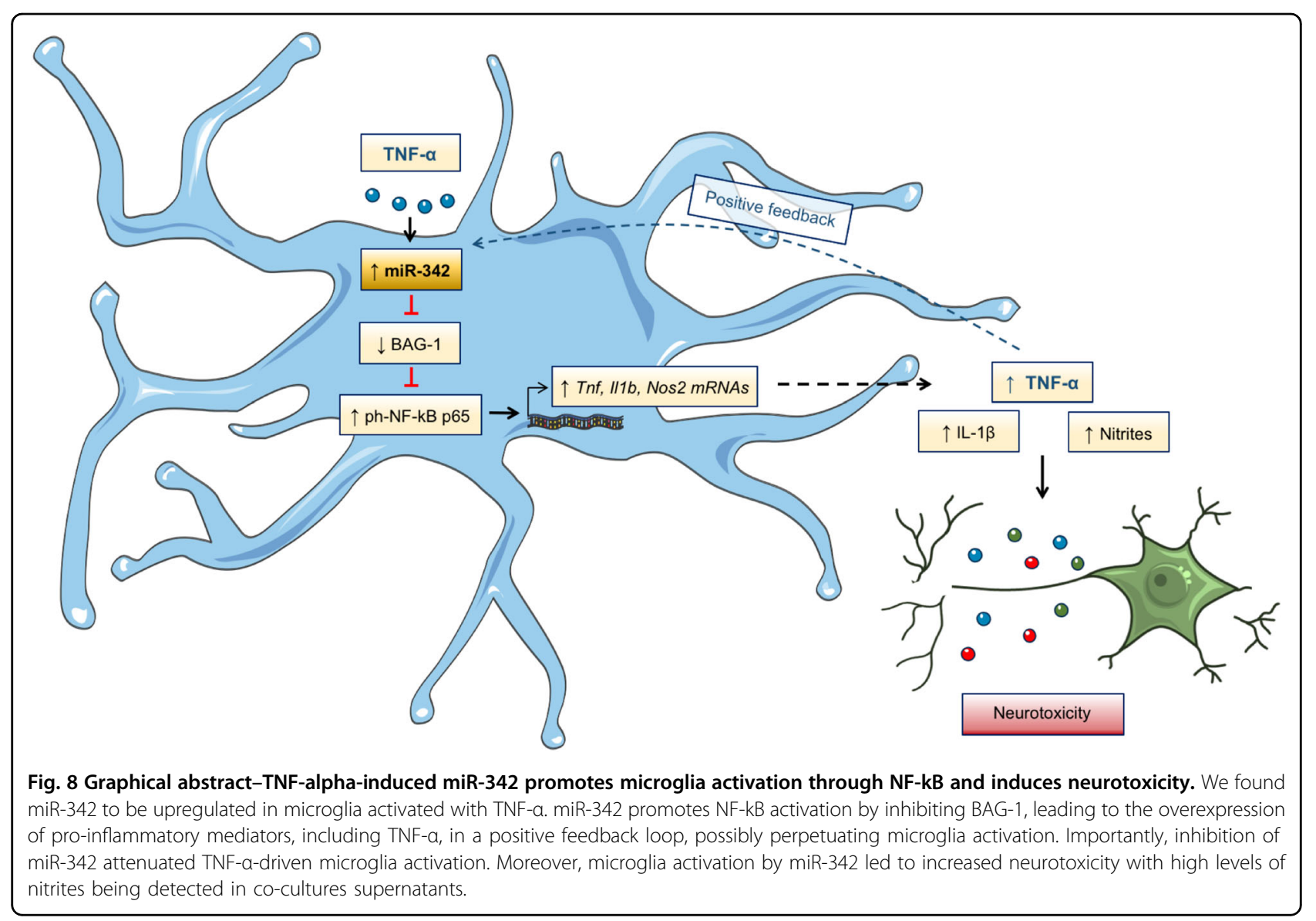

cells. The authors reported that mouse dendritic cells deficient in either HSP70 or BAG-1 had more nuclear p65 and produced more pro-inflammatory cytokines than did wild-type dendritic cells ${ }^{29}$, implicating BAG-1 as a negative regulator of pro-inflammatory NF-kB signaling in dendritic cells. Our study shows, for the first time, that BAG-1 is a mediator of NF-kB signaling in microglia, and that BAG-1 protein levels are regulated by miR-342.

Nevertheless, it is important to note that miRNAs can target multiple transcripts, as such we do not exclude that other proteins with relevance for microglia activation, can also be affected by miR-342 overexpression. Specifically, DAVID functional annotation analysis revealed that inflammation-related processes were the most upregulated biological functions associated with miR-342 overexpression (Fig. 5c). Particularly, FADD and MIF expression regulation by miR-342 should be further explored, as they are known to potentiate NF-kB activation and pro-inflammatory cytokines production ${ }^{40,41}$.

The deleterious effects of excessive production of cytokines and nitric oxide species in neurons have been extensively studied ${ }^{32,37}$. For instance, TNF- $\alpha$ can potentiate glutamate-mediated cytotoxicity by two complementary mechanisms: indirectly, by inhibiting glutamate transport on astrocytes, and directly, by stimulating extensive microglial and/or astrocyte glutamate release in an autocrine manner. This disturbs the balance of excitation and inhibition, resulting in a higher synaptic excitatory/inhibitory ratio-excitoneurotoxicity ${ }^{37,42,43}$. On the other hand, microglial derived nitric oxide inhibits neuronal respiration, resulting in glutamate release and subsequent excitotoxicity ${ }^{44,45}$. As such, to address the effect of miR-342 overexpression in the cross-talk with neurons, we co-cultured microglia with hippocampal neurons. Interestingly, we found that, in both TNF- $\alpha$ stimulated and miR-342 transfected microglia, neuron viability was drastically affected compared with the controls. We hypothesize that this may be owing to an increased production of the pro-inflammatory cytokines IL-1 $\beta$ and TNF- $\alpha$ (Results section 3 ), but also owing to increased levels of nitrites detected in the supernatants of these co-cultures. Not surprisingly, and given the upregulation of Nos2, supernatants of TNF- $\alpha$ stimulated microglia show an increased level of nitrites. Moreover, miR-342 overexpression also induced neuronal cell death, revealing that miR-342 impacts microglia activation as well as its cross-talk with neurons. In the current work, the possibility that these findings are cell line specific 
cannot be completely ruled out. It would be interesting to pursue this work in primary microglia cells, and further to perform in vivo administration of miR-342 targeted to microglia, to clarify its impact on the cross-talk between microglia and neurons, as well as the repercussion on animals' behavior, particularly in animal models of neuroinflammation.

These findings support miR-342 as potential target to resolve neuroinflammation, characterized by increased levels of TNF- $\alpha$, sustained microglia activation, and often associated with the development of neurological and psychiatric disorders.

\section{Acknowledgements}

We would like to thank Dr. João Relvas laboratory for the help with N9 microglia cell culture; Dr. Sofia Lamas for the guidance on the animal welfare and support with animal experiments (Animal facility, i3S); and to LC Sciences for the miRNA microarray data and analysis. The mass spectrometry technique was performed by Hugo Osório at the i3S Proteomics Scientific Platform with support from the Portuguese Mass Spectrometry Network, integrated in the National Roadmap of Research Infrastructures of Strategic Relevance (ROTEIRO/0028/2013; LISBOA01-0145-FEDER-022125). This work was funded by project NORTE-01-0145FEDER-000012, supported by Norte Portugal Regional Operational Programme (NORTE 2020), under the PORTUGAL 2020 Partnership Agreement, through the European Regional Development Fund (ERDF). J.P.B. and J.B. are supported by FCT-Fundação para a Ciência e Tecnologia, through BiotechHealth PhD program fellowship (PD/BD/135490/2018) and Areas of Basic and Applied Biology PhD program fellowship (PD/BD/135450/2017), respectively.

\section{Author details}

'i3S - Instituto de Investigação e Inovação em Saúde, University of Porto, Rua Alfredo Allen 208, 4200-135 Porto, Portugal. ${ }^{2}$ INEB - Instituto de Engenharia Biomédica, University of Porto, Rua Alfredo Allen 208, 4200-135 Porto, Portugal. ${ }^{3}$ ICBAS - Instituto de Ciências Biomédicas Abel Salazar, University of Porto, Rua de Jorge Viterbo Ferreira 228, 4050-313 Porto, Portugal. ${ }^{4}$ IBMC - Instituto de Biologia Molecular e Celular, University of Porto, Rua Alfredo Allen 208, 4200135 Porto, Portugal. ${ }^{5}$ ESS - Escola Superior de Saúde, Instituto Politécnico do Porto, Rua Dr. António Bernardino de Almeida 400, 4200-072 Porto, Portugal

\section{Conflict of interest}

The authors declare that they have no conflict of interest.

\section{Publisher's note}

Springer Nature remains neutral with regard to jurisdictional claims in published maps and institutional affiliations.

Supplementary Information accompanies this paper at (https://doi.org/ 10.1038/s41419-020-2626-6).

Received: 19 September 2019 Revised: 7 April 2020 Accepted: 5 May 2020 Published online: 02 June 2020

\section{References}

1. Miller, A. H. \& Raison, C. L. The role of inflammation in depression: from evolutionary imperative to modern treatment target. Nat. Rev. Immunol. 16, 22-34 (2016)

2. Yirmiya, R.r Rimmerman, N. \& Reshef, R. Depression as a microglial disease. Trends Neurosci. 38, 637-658 (2015)

3. Ginhoux, F. et al. Fate mapping analysis reveals that adult microglia derive from primitive macrophages. Science 330, 841-845 (2010).

4. Thion, M. S., Ginhoux, F. \& Garel, S. Microglia and early brain development: an intimate journey. Science 362, 185-189 (2018).
5. Ransohoff, R. M. \& Perry, V. H. Microglial physiology: unique stimuli, specialized responses. Annu. Rev. Immunol. 27, 119-145 (2009).

6. Dheen, S. T., Kaur, C. \& Ling, E. A. Microglial activation and its implications in the brain diseases. Curr. Med. Chem. 14, 1189-1197 (2007).

7. Perry, V. H. \& Holmes, C. Microglial priming in neurodegenerative disease. Nat. Rev. Neurol. 10, 217-224 (2014).

8. Welser-Alves, J. V. \& Milner, R. Microglia are the major source of TNF-a and TGF- $\beta 1$ in postnatal glial cultures; regulation by cytokines, lipopolysaccharide, and vitronectin. Neurochem. Int. 63, 47-53 (2013).

9. Riazi, K. et al. Microglial activation and TNFa production mediate altered CNS excitability following peripheral inflammation. Proc. Natl Acad. Sci. 105, 17151 (2008).

10. Sedger, L. M. \& McDermott, M. F. TNF and TNF-receptors: from mediators of cell death and inflammation to therapeutic giants - past, present and future. Cytokine Growth Factor Rev. 25, 453-472 (2014).

11. Golan, H., Levav, T., Mendelsohn, A. \& Huleihel, M. Involvement of tumor necrosis factor alpha in hippocampal development and function. Cereb. Cortex 14, 97-105 (2004)

12. Block M. L., Zecca L., Hong J. S. Microglia-mediated neurotoxicity: uncovering the molecular mechanisms. Nat. Rev. Neurosci. 8, 57-69 (2007).

13. Kuno, R. et al. Autocrine activation of microglia by tumor necrosis factor-alpha. J. Neuroimmunol. 162, 89-96 (2005).

14. Kolshus, E., Dalton, V. S., Ryan, K. M. \& McLoughlin, D. M. When less is more-microRNAs and psychiatric disorders. Acta Psychiatr. Scand. 129, 241-256 (2014).

15. Geaghan, M. \& Cairns, M. J. MicroRNA and posttranscriptional dysregulation in psychiatry. Biol. Psychiatry 78, 231-239 (2015).

16. Almeida, M. I., Reis, R. M. \& Calin, G. A. MicroRNA history: discovery, recent applications, and next frontiers. Mutat. Res. 717, 1-8 (2011).

17. Almeida, M. I., Reis, R. M. \& Calin, G. A. Decoy activity through microRNAs: the therapeutic implications. Expert Opin. Biol. Ther. 12, 1153-1159 (2012).

18. Almeida, M. I. et al. Strand-specific miR-28-5p and miR-28-3p have distinct effects in colorectal cancer cells. Gastroenterology 142, 886-896 (2012).

19. Almeida, M. I. \& Calin, G. A. The miR-143/miR-145 cluster and the tumor microenvironment: unexpected roles. Genome Med. 8, 29 (2016).

20. Bras, J. P. et al. miR-195 inhibits macrophages pro-inflammatory profile and impacts the crosstalk with smooth muscle cells. PloS ONE 12, e0188530 (2017).

21. Alexander, M. et al. Exosome-delivered microRNAs modulate the inflammatory response to endotoxin. Nat. Commun. 6, 7321 (2015).

22. Tamashiro, T. T., Dalgard, C. L. \& Byrnes, K. R. Primary microglia isolation from mixed glial cell cultures of neonatal rat brain tissue. J. Vis, Exp. 66, e3814 (2012)

23. Seibenhener, M. L. \& Wooten, M. W. Isolation and culture of hippocampal neurons from prenatal mice. J. Vis. Exp. 65, 3634 (2012).

24. Bustin, S. A. et al. The MIQE guidelines: minimum information for publication of quantitative real-time PCR experiments. Clin. Chem. 55, 611-622 (2009).

25. Kozomara, A., Birgaoanu, M. \& Griffiths-Jones, S. miRBase: from microRNA sequences to function. Nucleic Acids Res. 47, D155-d162 (2019).

26. Silva, A. M. et al. Resveratrol as a natural anti-tumor necrosis factor-alpha molecule: implications to dendritic cells and their crosstalk with mesenchymal stromal cells. PloS ONE 9, e91406 (2014).

27. Crowley L. C., Marfell B. J., Waterhouse N. J. Analyzing cell death by nuclear staining with Hoechst 33342. Cold Spring Harb. Protoc. 2016, https://doi.org/ 10.1101/pdb.prot087205 (2016).

28. Huang da, W., Sherman, B. T. \& Lempicki, R. A. Systematic and integrative analysis of large gene lists using DAVID bioinformatics resources. Nat. Protoc. 4, 44-57 (2009).

29. Tanaka, T., Shibazaki, A., Ono, R. \& Kaisho, T. HSP70 mediates degradation of the p65 subunit of nuclear factor kappaB to inhibit inflammatory signaling. Sci. Signal. 7, ra119 (2014)

30. Miller, A. H., Haroon, E., Raison, C. L. \& Felger, J. C. Cytokine targets in the brain: impact on neurotransmitters and neurocircuits. Depress. Anxiety 30, 297-306 (2013).

31. Zhang, L., Zhang, J. \& You, Z. Switching of the microglial activation phenotype is a possible treatment for depression disorder. Front. Cell. Neurosci. 12, 306-306. (2018).

32. Brown G. C., Neher J. J. Inflammatory neurodegeneration and mechanisms of microglial killing of neurons. Mol. Neurobiol. 41, 242-247 (2010).

33. Streit, W. J., Mrak, R. E. \& Griffin, W. S. T. Microglia and neuroinflammation: a pathological perspective. J. Neuroinflamm 1, 14-14 (2004). 
34. Stuart, M. J. \& Baune, B. T. Chemokines and chemokine receptors in mood disorders, schizophrenia, and cognitive impairment: a systematic review of biomarker studies. Neurosci. Biobehav. Rev. 42, 93-115 (2014).

35. Rosenblat, J. D., Cha, D. S., Mansur, R. B. \& Mclntyre, R. S. Inflamed moods: review of the interactions between inflammation and mood disorders. Prog. Neuro-Psychopharmacol. Biol. Psychiatry 53, 23-34 (2014).

36. Raison, C. L., Capuron, L. \& Miller, A. H. Cytokines sing the blues: inflammation and the pathogenesis of depression. Trends Immunol. 27, 24-31 (2006).

37. Olmos, G. et al. Tumor necrosis factor alpha: a link between neuroinflammation and excitotoxicity. Mediators Inflamm. 2014, 12 (2014).

38. Gotz, R. et al. Bag1 is essential for differentiation and survival of hematopoietic and neuronal cells. Nat. Neurosci. 8, 1169-1178 (2005).

39. Takayama, S. et al. Cloning and functional analysis of BAG-1: a novel BCl-2binding protein with anti-cell death activity. Cell 80, 279-284 (1995).

40. Kreuz, S. et al. NFkappaB activation by Fas is mediated through FADD, caspase-8, and RIP and is inhibited by FLIP. J. Cell Biol. 166, 369-380 (2004).
41. Zhang, Y. et al. Inhibition of macrophage migration inhibitory factor (MIF) tautomerase activity suppresses microglia-mediated inflammatory responses. Clin. Exp. Pharmacol. Physiol. 43, 1134-1144 (2016).

42. Takeuchi, $\mathrm{H}$. et al. Tumor necrosis factor-alpha induces neurotoxicity via glutamate release from hemichannels of activated microglia in an autocrine manner. J. Biol. Chem. 281, 21362-21368 (2006).

43. Bezzi, P. et al. CXCR4-activated astrocyte glutamate release via TNFalpha: amplification by microglia triggers neurotoxicity. Nat. Neurosci. 4, 702-710 (2001).

44. Boje, K. M. \& Arora, P. K. Microglial-produced nitric oxide and reactive nitrogen oxides mediate neuronal cell death. Brain Res. 587, 250-256 (1992).

45. Bal-Price, A. \& Brown, G. C. Inflammatory neurodegeneration mediated by nitric oxide from activated glia-inhibiting neuronal respiration, causing glutamate release and excitotoxicity. J. Neurosci. 21, 6480-6491 (2001). 